\title{
Tratamiento económico y modelización del cuidado
}

\author{
Economic treatment and modeling of care
}

\author{
Montserrat Díaz-Fernández / mdiaz@uniovi.es \\ https://orcid.org/0000-0002-3864-0041 \\ Mar Llorente-Marrón / mmarron@uniovi.es \\ https://orcid.org/0000-0001-7409-1695 \\ Sandra Dema-Moreno / demasandra@uniovi.es \\ https://orcid.org/0000-0003-1845-5962 \\ Universidad de Oviedo, España
}

\begin{abstract}
Care is an expansive concept that includes the set of activities aimed at ensuring the survival and well-being of the population. This article aims to analyze and characterize care as an economic good whose provision is made through the market, public administration and households, with different implications and treatments in these three spheres. To this end, once the problem posed by this good from a multidisciplinary perspective is presented, we will focus on the characteristics that economic theory gives to economic goods and discuss how each of them manifests in the case of care. We will also analyze care's supply and demand curves, the agents involved and its market equilibrium. The economic description leads us to consider that the behavior of this market deviates substantially from the characteristics of the so-called perfect competition market and it also indicates the need to carry out a social reorganization of care.
\end{abstract}

Key words: care, economic analysis, economic good, care market, demand and supply of care.

Resumen: El cuidado es un concepto expansivo que incluye el conjunto de actividades tendentes a garantizar la supervivencia y el bienestar de la población. Este artículo se propone analizar y caracterizar el cuidado como bien económico cuya provisión se realiza a través del mercado, administración pública y hogar, con implicaciones y tratamientos diferentes en estos tres ámbitos. Para ello, una vez presentada la problemática planteada desde una perspectiva pluridisciplinar, nos centraremos en las características que la teoría económica otorga a los bienes económicos y discutiremos cómo cada una de ellas se manifiesta en el cuidado. También se analizarán sus curvas de oferta y demanda, los agentes implicados y el equilibrio del mercado. La descripción económica realizada nos conduce a considerar que el comportamiento de este mercado es claramente imperfecto y además nos indica la necesidad de efectuar una reorganización social del cuidado.

Palabras clave: cuidado, análisis económico, bien económico, mercado de cuidado, demanda y oferta de cuidado. 


\section{Introducción}

El cuidado es un concepto expansivo en el que se incluye el conjunto de actividades tendentes a garantizar la supervivencia y el bienestar de la población en el desempeño de su actividad diaria. Las investigaciones acerca del cuidado, sin embargo, no han formado parte de la disciplina económica hasta fechas recientes, con el desarrollo de planteamientos vinculados con la incorporación de la perspectiva de género en esta área de conocimiento.

Dichas investigaciones han puesto de manifiesto que el cuidado ha sido una actividad tradicionalmente considerada femenina y provista casi en exclusiva por las mujeres al interior de los hogares. También han mostrado las consecuencias que genera la división sexual del trabajo sobre las relaciones de género y, particularmente, cómo el reparto desigual de las labores de cuidado y las tareas domésticas no remuneradas es un determinante importante de las desigualdades de género en el empleo.

Estos estudios han permitido, asimismo, que distintos estamentos de las instituciones públicas de las sociedades contemporáneas hayan puesto el foco en este fenómeno social y que la economía del cuidado haya pasado a formar parte de sus agendas. De hecho, aunque en la práctica todavía las mujeres asumen la mayor parte de la provisión del cuidado en nuestras sociedades, ya no se considera que sea una obligación exclusivamente suya ni que tal solución sea socialmente deseable (Tobío et al., 2010). Si bien el cuidado todavía no se ha conceptualizado como un derecho de ciudadanía, el reto en la actualidad consiste en lograr la responsabilidad social compartida y el reparto equitativo del cuidado entre la familia, el Estado y el mercado laboral (McMahon, 1999; Bonino, 2001).

En este artículo se pretende continuar en la línea de conocimiento abierta en el ámbito de la economía feminista, aportando un análisis del concepto de cuidado desde la teoría económica y, en concreto, ofreciendo una caracterización económica de dicho fenómeno. La necesidad de visibilizar en el análisis económico la faceta productiva del hogar, proveedor de bienes y servicios fundamentales para la supervivencia humana, hace de la modelización económica del cuidado una cuestión insoslayable. La definición del bien cuidado y el análisis de cómo funciona su mercado permitirá detectar las similitudes y diferencias respecto a otros bienes de la misma naturaleza, además de facilitar el abordaje de los problemas políticos que pueda suscitar, así como aquellas cuestiones vinculadas con sus necesidades de provisión y gestión o planificación, entre otras. Para ello, en primer lugar, presentaremos la problemática del cuidado desde una perspectiva pluridisciplinar y, posteriormente, 
abordaremos los distintos aspectos que componen el mercado de cuidado, la caracterización económica del bien, sus curvas de oferta y demanda, los agentes implicados y el equilibrio del mercado.

\section{Economía y cuidado en el pensamiento económico}

De acuerdo con la etimología, la palabra "economía” se remonta a las voces griegas oikos y nomo, casa y administración, respectivamente, y, por tanto, oikonomia significaría administración doméstica. No obstante, una breve mirada a la historia del pensamiento económico refleja la evolución del significado de este vocablo. A partir del siglo XVII se extiende su significado y comienza a hablarse de "economía política" para referirse al gobierno de una nación o región en su conjunto.

La publicación de La Riqueza de las Naciones de Adam Smith en 1776 marca el nacimiento del pensamiento económico moderno. La escuela clásica entiende la economía política como una disciplina que identifica las leyes que gobiernan la producción, el consumo y la distribución de los bienes y servicios que permiten la satisfacción de las necesidades de la población. El análisis de Adam Smith (2011) y David Ricardo (2003) se centró en estudiar el valor de los productos intercambiados en el sistema y lo que ello supone para las relaciones entre las personas en las sociedades desarrolladas.

La teoría del valor-trabajo limita el alcance del trabajo al mercado excluyendo de su definición todas aquellas labores no incorporadas en la órbita mercantil (Picchio, 1996 y 1999; Carrasco, 2011). Con posterioridad, en el siglo XIX, una nueva argumentación en torno al problema económico se identificó con la escasez y sus consecuencias, como un problema de deseos y posibilidades. La escuela marginalista sitúa como objeto de estudio el funcionamiento del mercado y su papel en la asignación de recursos escasos, manteniéndose en los márgenes de la economía monetizada. La separación de espacios entre el ámbito privado y el mercado invisibiliza aún más el trabajo del hogar o cuidado al no ser objeto de intercambio mercantil.

Desde la teoría neoclásica se parte de una visión diferente de lo que supone el trabajo doméstico, centrada en la elección individual, fundamento de la conocida como "Nueva Economía de la Familia”, elaborada básicamente en la Escuela de Chicago (Becker, 1987). Este planteamiento teórico rectifica la noción tradicional de familia, considerándola simultáneamente como una unidad de producción y consumo. Dicho enfoque se basa en el comportamiento racional del individuo, justificando la situación social de desigualdad de las mujeres en una elección. La tradicional división del trabajo entre hom- 
bres y mujeres, esto es, que ellos asuman mayoritariamente el trabajo remunerado y ellas el no remunerado se contempla desde esta perspectiva como una mera decisión racional por parte de los integrantes del hogar a la valoración que el mercado hace del tiempo y del trabajo que desarrollan cada uno de ellos (Gardiner, 1997).

La teoría feminista desafía todos estos presupuestos y enfatiza el valor del trabajo doméstico para la reproducción social (Benería, 1979; Peterson, 2009; Barker y Feiner, 2010; Bedford y Rai, 2010; Kofman, 2012; Carrasco, 2013). Desde estos planteamientos se critica tanto la visión economicista del trabajo, restringida a las actividades en las que media el mercado, como sus principios convencionales, integrando dentro de dicha noción todo lo referido al cuidado; esto es, aquella parcela específica del trabajo, relacionada con la calidad de vida de las personas y su cuidado personal directo o indirecto (Folbre, 2006).

De esta forma, se hace visible el fenómeno del cuidado, que había pasado desapercibido hasta ese momento para la economía, legitimándolo como objeto de estudio (Gutiérrez-Rodríguez, 2014). Las investigaciones desarrolladas muestran que los bienes y servicios adquiridos por los hogares en el mercado (comida, vestido, entre otros) no son suficientes para la reproducción de la fuerza de trabajo y es necesario realizar labores adicionales (cocina, lavado, planchado, limpieza, entre otras), destinadas a su transformación en bienes y servicios que permitan la satisfacción de las necesidades humanas y el mantenimiento del hogar.

Esta conceptualización del cuidado traspasa la frontera del trabajo no monetizado y cuestiona la división entre ambos tipos de trabajo: de mercado y de cuidado (Carrasco, 2013). De hecho, en momentos de expansión económica, es frecuente que se desplacen al mercado producciones que tradicionalmente se realizaban en el hogar (por ejemplo, la producción de ropa o comida) y, viceversa, en épocas de crisis la producción de determinados bienes y servicios se traslada al hogar (Carrasco, 2011).

Asimismo, estos estudios revelan, por un lado, la importancia vital de los cuidados en el sostenimiento de la vida humana, esto es, la necesidad de valorar esta actividad por sí misma y de reconocerla como el trabajo fundamental para que la vida continúe (Carrasco, 2013: 42) y, por otro, las dimensiones subjetivas, emocionales y relacionales del cuidado. El trabajo de cuidado de las personas se desarrolla en un espacio y en unas condiciones muy distintas a las del mercado, en el mismo intervienen actitudes y emociones que no pueden ser atendidas ni sustituidas por la producción de mercado (Himmelweit, 1995 y 2002; Folbre, 2006; Pérez-Orozco, 2004). 
A pesar de que el trabajo de cuidados contribuye económicamente y de forma significativa al bienestar personal y social, su dimensión productiva ha sido sistemáticamente ignorada en los cálculos oficiales del Producto Interior Bruto (PIB) hasta el desarrollo de las Cuentas Satélites de la Producción Doméstica (Himmelweit, 1995; Pérez-Orozco, 2004 y 2009) y de las Encuestas de Empleo del Tiempo, puestas en marcha en numerosos países. A pesar de que dichos intentos de valoración son claramente insuficientes, en tanto que no han sido desarrollados de forma sistemática y continuada, ni reflejan la complejidad del cuidado, en lo referente a las dimensiones subjetivas mencionadas antes sí han permitido visibilizar un fenómeno que hasta entonces había permanecido oculto.

Las citadas encuestas muestran que, por un lado, las mujeres asumen mayoritariamente el cuidado en nuestras sociedades, tanto al interior de sus propios hogares, de manera informal, como formalmente, cuando las tareas de cuidado se realizan de forma profesional, ya sea desde el mercado o a través del Estado (Rogero, 2010). A nivel mundial, las mujeres dedican una media de entre tres y seis horas diarias a las tareas de cuidado no remuneradas, mientras que los varones dedican entre media hora y dos horas (Ferrant et al., 2014); si bien estas diferencias oscilan por regiones y modelos de desarrollo y bienestar.

Por otro lado, se observa que la asunción mayoritaria de las tareas domésticas por parte de las mujeres reduce el tiempo libre del cual disponen, impidiéndoles participar en el empleo en condiciones de igualdad con los varones y limitando su participación en otros ámbitos sociales, al tiempo que las empobrece, pues los cuidados realizados al interior del hogar se prestan de forma gratuita. Si nos referimos al cuidado formal, que se lleva a cabo a través del Estado y/o del mercado, también se observa en la mayor parte de los países que las mujeres se concentran en sectores feminizados y vinculados al cuidado, como la salud, la educación, los servicios sociales o el servicio doméstico, entre otros.

Esta segregación ocupacional conlleva además importantes consecuencias sociales para las mujeres por la escasa valoración de las tareas de cuidado, que se traduce en bajos salarios y condiciones laborales muy precarias, fundamentalmente en el caso del servicio doméstico. La economía del cuidado se une a los debates acerca de protección social y disponibilidad de servicios de cuidado; se extiende más allá de los hogares incorporando a la familia, el Estado, el mercado y los sectores sin fines de lucro (Folbre, 2006; Himmelweit, 2007). 
Pero tal vez la mayor aportación de estos planteamientos a la economía consista en replantear el propio objeto de estudio de esta disciplina. Al poner de manifiesto que el trabajo de cuidado constituye un aspecto esencial para el bienestar de la población, el foco de la economía, del capital y la producción mercantil se traslada a las personas y la satisfacción de sus necesidades (Picchio, 2001 y 2009; Enríquez, 2012), lo cual supone un cambio de paradigma de consecuencias teóricas y metodológicas trascendentales.

En definitiva, el conocimiento y la visualización del alcance real de la actividad que genera el desempeño del cuidado han estado acompañados de cambios conceptuales tendentes a delimitar el significado y las dimensiones básicas de la economía del cuidado, así como la manera de organizarla socialmente (Esquivel, 2011). Dicho cambio conceptual genera importantes consecuencias metodológicas; el análisis del cuidado no remunerado en el seno de los hogares, así como del provisto a través del mercado, el Estado y otras organizaciones comunitarias y sociales, permite identificar y enfatizar aquellos elementos del mismo que producen o contribuyen a producir valor económico. Y no sólo eso, la incorporación de estas actividades a las cuentas nacionales permite visibilizar la faceta productiva del hogar, el trabajo no remunerado, y sus conexiones estructurales con las demás actividades socioeconómicas desarrolladas por la población.

Con este artículo buscamos contribuir al proceso de reflexión acerca del cuidado, planteando dos cuestiones que aún no han sido abordadas en nuestra disciplina: en primer lugar, una caracterización económica de dicho bien $y$, en segundo lugar, el funcionamiento de su mercado. Se pretende llevar a cabo dicha caracterización económica, identificando y destacando aquellos elementos del cuidado que producen o contribuyen a producir valor económico, con el fin de favorecer la visibilidad de las relaciones entre mercado y hogar, y conocer las formas de distribución del tiempo, el trabajo, remunerado y no remunerado y la renta, que se deriva de la realización del trabajo mercantil.

\section{Caracterización económica del cuidado}

Desde el punto de vista económico, el cuidado es un concepto expansivo en el que se incluye el conjunto de actividades tendentes a incrementar el nivel de vida de la población en el desarrollo de su actividad diaria. En su definición abarca tanto la acción de cuidar a otros como el autocuidado, e incorpora la perspectiva de quienes proporcionan la provisión del recurso y la de quienes la reciben. La acción de cuidar comprende atención, control, com- 
pañía, cuidados sanitarios y proporciona apoyo emocional y social a las personas (García-Calvente et al., 2004; Gutiérrez-Rodríguez, 2014). Por tanto, es un trabajo con una dimensión no sólo material y económica, sino también psicológica y afectiva (Batthyamy, 2009).

El cuidado es un un recurso limitado y escaso susceptible de ser adquirido en el mercado previa satisfacción de su precio (Esquivel, 2011). Es un bien económico, puesto que le caracteriza la escasez, esto es, la demanda supera a la oferta. Las necesidades de cuidado son crecientes e ilimitadas (demanda ilimitada) y los recursos son finitos (oferta limitada); por ello, es inevitable adjudicar esos recursos a diversas actividades en competencia.

A continuación señalamos sus características para, posteriormente, analizar los agentes económicos que intervienen en su provisión y en el funcionamiento de su mercado.

\section{Caracteristicas del cuidado como bien económico}

El cuidado satisface un conjunto amplio de características comunes a otros bienes de naturaleza económica, aunque con una intensidad diferente (DíazFernández y Llorente-Marrón, 2011), lo cual le confiere un estatus especial. Metodológicamente se identifica con una variable de flujo, pues se mide por unidad o periodo. De hecho, podemos definir el cuidado como el flujo de bienes y servicios generados en un periodo por el Estado, el mercado y la familia, con el objetivo de conseguir cotas de bienestar individual y social. A este respecto, los estudios de uso del tiempo permitieron contabilizar la cantidad de éste que dedican mujeres y hombres al cuidado dentro del hogar. Ello posibilita no sólo conocer la magnitud de las desigualdades derivadas de la división sexual del trabajo, sino también fomenta la medición de su valor económico, a través de algunos intentos de incorporar las actividades de cuidado a las cuentas nacionales (Carrasco, 2011).

Teniendo en cuenta su funcionabilidad o destino, el cuidado constituye un bien económico de inversión tanto para la persona como para la sociedad, ya que su resultado va dirigido al incremento de la producción y, por tanto, de la renta y también del ahorro en los hogares. La investigación empírica sugiere que la valoración del trabajo que no es de mercado tiene un fuerte efecto igualador en el ingreso y consumo prolongado (Frick et al., 2009; Frazis y Stewart, 2011; Folbre 2015). Asimismo, constituye un importante ahorro para el hogar, en tanto que todos aquellos bienes y servicios que se producen en su interior evita tener que comprarlos a precio de mercado (Dema y Díaz, 2014). 
Es un bien necesario que satisface necesidades básicas del ciclo vital e imprescindible en el proceso de supervivencia. Las necesidades de cuidado se generan individual y socialmente; forman parte del sistema económico en el cual se ubican y de la sociedad en su conjunto, que condiciona la disponibilidad y necesidad de dicho bien. A lo largo del ciclo de vida, las personas consumen cuidados con distinta intensidad y modalidad, ya sea de manera autónoma o con mayor o menor dependencia de otras (Izquierdo, 2003).

La naturaleza compleja y heterogénea son rasgos que también están presentes en el cuidado. Las emociones y afectividades personales forman parte de las actividades de este tipo que se llevan a cabo en el hogar, como diversas economistas se han encargado de mostrar (Folbre, 1995 y 2006; Himmelweit, 1995 y 2002; Pérez-Orozco, 2004). El cuidado siempre presenta una doble cara: alguien que recibe y alguien que da, y constituye un bien diferente según su localización, las necesidades por satisfacer y, en su caso, la remuneración que genera. Desde esta perspectiva, el cuidado incluye tanto la atención a niños, como el autocuidado y el correspondiente a las personas adultas dependientes.

Otro rasgo relevante de los cuidados es su universalidad, los seres humanos precisan de actividades que les permitan sobrevivir en distintas etapas de su ciclo vital y con intensidades diferentes. Los cuidados son necesarios para la población, de ahí la preocupación del sector público por la eficiencia en las decisiones asignativas y las desigualdades en la distribución del consumo de los mismos. No obstante, el alcance de las políticas públicas en esta materia es diferente en función de la condición social y de la localización territorial. En muchos países, su evolución demográfica, tendente al envejecimiento de la población, requiere de forma inevitable de una respuesta del sector público a las necesidades de cuidados, pero ésta recae fundamentalmente en los hogares, actuando la provisión pública como mero complemento en caso de ser necesario.

Es un bien importante; el elevado precio del cuidado en términos sociales hace del recurso para la financiación del entorno familiar una cuestión muchas veces ineludible, constituyendo una decisión económica y social relevante en el ciclo de vida (Yoon, 2014). El cuidado es costoso, pero paradójicamente no siempre bien valorado en términos económicos. De hecho, con frecuencia se percibe a las personas cuidadoras como mano de obra improductiva y/o no cualificada (Gutiérrez-Rodríguez, 2014).

Según la Organización Internacional del Trabajo (OIT, 2016), la mayoría de las personas cuidadoras son mujeres (82\%). Precisamente la feminización de los cuidados representa para la economía feminista una de las causas 
de que no se le considere en las distintas teorías económicas. Folbre (1995) señala la necesidad de tomar en cuenta el papel determinante de las relaciones de género en las actividades del hogar y del mercado, lo que hace del precio sombra, esto es, el coste de oportunidad de producir un bien o servicio, un elemento decisivo en la modelización económica de dicho bien o servicio.

La economía feminista contribuye conceptual y metodológicamente a visibilizar el rol de este trabajo de cuidado en el funcionamiento de la economía, entendiendo que los hogares son entes complejos en cuyo interior se negocia implícita o explícitamente la división del trabajo entre sus miembros. Tiempo, dinero y servicios son los componentes del cuidado. Su organización establece distintas relaciones de género en la unidad familiar que favorecen la consolidación de los modelos de provisión de cuidado (Ellingstaeter, 1999: 590).

En la teoría económica se diferencia entre bienes muebles e inmuebles, considerándose un bien mueble aquel que puede ser trasladado de un lugar a otro sin pérdida de su valor o utilidad, respondiendo a las necesidades del entorno, nacional o internacional. Desde dicha perspectiva, el cuidado se configura como un bien mueble, cuando es emprendido en el mercado, e inmueble, si es realizado en el ámbito doméstico.

El cuidado es además un bien duradero, es decir, un bien de consumo prolongado en el ciclo de vida del individuo. El aumento de la esperanza de vida, consecuencia del envejecimiento de la población y la reducción de la natalidad, provoca un incremento incesante e insatisfecho de cuidados, relacionado con la problemática de la dependencia (Durán, 2006; RodríguezCabrero, 2007). Estas necesidades de cuidado de la población sitúan a las sociedades contemporáneas ante uno de los grandes retos de nuestro tiempo: la solución colectiva de esta problemática, que por diversas razones ya no puede resolverse de forma única en el seno familiar. Autoras como Mary Daly y Jane Lewis entienden que el Estado debe proveer a la ciudadanía de mecanismos para el cuidado cotidiano durante los momentos del ciclo vital en los cuales se carece de autonomía personal (Lewis, 2007): el denominado social care (Daly y Lewis, 2000).

No obstante, esta demanda de cuidado como derecho de ciudadanía no tiene aún sustento jurídico en los Estados de bienestar. De hecho, en la mayor parte de las legislaciones el derecho y las responsabilidades del cuidado se agotan en la atención de las criaturas por parte de sus padres y, sobre todo, de sus madres, sin que encontremos previsión alguna respecto al papel que pueda jugar el resto de la sociedad. Tampoco aparecen fórmulas que garanticen el derecho a cuidar o ser cuidado del resto de las personas, salvo en algunos 
casos el de personas adultas dependientes con las que también se tengan vínculos familiares. Todas estas características confieren al bien cuidado de un estatus característico en la economía, aspecto que se visibiliza en el funcionamiento de su mercado.

\section{El mercado de cuidado}

El individuo consume cuidados en momentos diferentes de su vida, distribuyendo su renta entre estos bienes y servicios y el resto. La asignación de los recursos se realiza a través del precio, resultado de las distintas negociaciones entre quienes ofertan y demandan los diferentes bienes y servicios.

Los mercados perfectos se caracterizan por ser modelos ideales en los que todas las personas participantes tienen el mismo grado de información. Un fallo de los mercados lo constituye la información imperfecta, presente en muchos de ellos, y entre éstos en el que se intercambian unidades y servicios de cuidado. En términos formales esto significa la ausencia de un conocimiento completo acerca de las características del cuidado, precios, necesidades, etc. Quienes demandan y ofertan cuidados en muchos casos no son plenamente conscientes de las características del bien objeto de transacción. La mayoría de la población no tiene la capacitación necesaria para atender a un gran dependiente; la brecha entre el conocimiento de la persona cuidadora y la dependiente es muy amplia.

El cuidado es un bien normal, su curva de demanda es decreciente; esto es, establece una relación inversa entre precios y unidades de consumo. Por el contrario, la curva de oferta de cuidado es creciente. Un aumento del precio generará incentivos en las unidades de producción para incrementar el número de unidades producidas de cuidado. El equilibrio se alcanza en la intersección de ambas funciones, destacando la estabilidad como rasgo distintivo de un mercado de competencia perfecta.

En la demanda se distinguen dos componentes fundamentalmente: la demanda a largo plazo, o demanda potencial determinada por variables de naturaleza sociodemográfica, y la de corto plazo, en la cual a dichos factores se suman los de naturaleza económica y las necesidades coyunturales. Los cambios en el comportamiento de dichos factores no repercuten en el precio del cuidado, traduciéndose en desplazamientos de la curva de demanda.

En relación con la oferta, la provisión se localiza en el ámbito del mercado (salud, educación, servicios personales, servicio doméstico, etc.), Estado (políticas públicas en el área de la salud, educación, servicios sociales, regulación laboral, etc.) y hogar, y, en este caso, la tarea es realizada generalmente 
por mujeres. La disponibilidad de recursos básicos, así como las condiciones económicas y las políticas públicas desempeñan un papel relevante. Variaciones en su dinámica generarán desplazamientos de la función de oferta y no variaciones en el precio del servicio.

En el largo plazo, el mercado debe tender al equilibrio, que se alcanza cuando la oferta de cuidado se ajusta con la demanda potencial (Figura $1^{1}$ ). Es preciso destacar que no toda la dotación del trabajo disponible está mercantilizada. Hay personas, principalmente mujeres, que se concentran de tiempo completo en el trabajo doméstico y de cuidado o desarrollan estrategias que combinan dedicación parcial de trabajo en el mercado con el no remunerado (Gutiérrez-Rodríguez, 2014).

A partir de un bien anómalo como el descrito, es lógico deducir que la oferta y la demanda presenten comportamientos desviados en relación con los que se adoptan en otros mercados y que ello perfile un funcionamiento del mercado no regular. El comportamiento real se desvía sustancialmente de las características propias del denominado mercado de competencia perfecta. Desequilibrio en el peso de oferentes y demandantes, equilibrio de mercado, asimetrías y externalidades significativas en el consumo, proporción reducida de unidades de cuidado en el mercado, entre otros factores, constituyen rasgos de un mercado imperfecto y segmentado, el cual impide que el mecanismo de precios descrito sea óptimo en la asignación de recursos.

\section{Conclusiones}

En este artículo se ha planteado y discutido la problemática del cuidado desde una perspectiva económica, abordando los distintos aspectos que componen el mercado de cuidado, con el fin de favorecer la visibilidad de las relaciones entre mercado y hogar. El análisis y conocimiento exhaustivo del papel funcional del cuidado cubre una desprovista dimensión del análisis económico que muestra la enorme relevancia social y económica de las actividades de este tipo. De hecho, sin el desempeño de las tareas del cuidado, la fuerza de trabajo y la producción se verían seriamente afectadas y la supervivencia de la vida humana no sería viable.

Desde la perspectiva económica, el cuidado es un recurso limitado y escaso, susceptible de ser adquirido en el mercado previa satisfacción de su precio, que comparte un conjunto amplio de características comunes con otros bienes de naturaleza económica, aunque con una intensidad diferente (Díaz-Fernández y Llorente-Marrón, 2011). Metodológicamente se identifica

1 Esta figura se encuentra en el Anexo, al final del presente artículo (Nota del editor). 
con una variable flujo y constituye un bien económico de inversión tanto para la persona como para la sociedad, pues su resultado se destina al incremento de la producción y, por consiguiente, de la renta y/o del ahorro al interior de los hogares.

Entre sus características sobresale su carácter de bien necesario e imprescindible en el proceso de supervivencia, su naturaleza compleja y heterogénea, así como su universalidad e importancia. Se configura como un bien mueble cuando es llevado a cabo en el mercado, e inmueble, si es realizado en el ámbito doméstico, y tiene carácter de bien duradero, ya que su consumo se efectúa en distintas y prolongadas etapas de la vida de las personas.

Teniendo en cuenta que el cuidado es un bien normal, su curva de demanda establece una relación inversa entre precios y unidades de consumo; mientras que la curva de oferta es creciente. La estabilidad constituye un rasgo distintivo de un mercado de competencia perfecta, y en el largo plazo, el mercado debe tender al equilibrio, que se alcanza cuando la oferta de cuidado se ajusta con la demanda potencial.

La descripción económica realizada del bien cuidado nos conduce a considerar que el comportamiento de su mercado se desvía sustancialmente de las características propias del denominado mercado de competencia perfecta. Mercado asimétrico y con fuertes externalidades, escasez de oferta remunerada, distinta ponderación de oferentes y demandantes, entre otros factores, son rasgos distintivos de un mercado, el de los cuidados, imperfecto y segmentado, que impiden que el mecanismo de precios sea óptimo en la asignación de recursos.

Por último, una cuestión fundamental es la relativa a la justificación en la cual se sustenta la modelización del mercado del cuidado como competencia perfecta. En la práctica se suele considerar como tal, tomando en cuenta su dimensión. Metodológicamente, la modelización del mercado como competencia perfecta significa que cualquier actuación pública que se adopte en este mercado satisface las condiciones de eficiencia y equidad. Dicha modelización tutela las actuaciones públicas en relación con la eficiencia y equidad. No obstante, la mayoría de las sociedades han mostrado una escasa presencia del Estado en la satisfacción de las necesidades y demandas de cuidado de la ciudadanía.

El incremento de la eficiencia, del grado de equidad en la distribución de la renta y la atenuación de los ciclos económicos son los argumentos habitualmente utilizados para justificar la intervención pública en la actividad económica. Desde este punto de vista, el cuidado es un bien preferente o esencial, por lo tanto facilitar el acceso al mismo a las familias más desfavore- 
cidas en términos económicos representa uno de los argumentos de la intervención pública: reducir las desigualdades existentes e incrementar el grado de equidad. Su consideración como bien necesario y la existencia de fallos en el mercado es otra de las razones para dicha intervención.

Considerarlo como bien económico permite a la Administración Pública disponer de criterios plurales para justificar su intervención. Dado que el mercado en el cual se intercambia el cuidado es claramente imperfecto, la eficiencia adquiere protagonismo cuando la Administración Pública se plantea como objeto la consecución de determinadas cotas de eficiencia. En síntesis, actitud paternalista, intento de mejora del estado de bienestar, visibilidad de las actuaciones, información asimétrica, externalidades o necesidades preferentes son algunas razones que podrían justificar dicha intervención, no exenta de dificultades y problemas.

Todas estas características económicas del bien y del mercado de cuidados, puestas de manifiesto en el presente artículo, nos llevan a una última reflexión relacionada con el contexto social donde se lleva a cabo el trabajo doméstico y de cuidados. En las últimas décadas se observan algunas tendencias como la incorporación masiva de las mujeres al empleo, el descenso en los niveles de fecundidad y un incremento progresivo de la esperanza de vida, lo cual hace urgente una reorganización social del cuidado.

Hoy por hoy, el modelo de relaciones laborales, con jornadas interminables, no permite satisfacer el cuidado de la población. Los diferentes regímenes de bienestar todavía no han incorporado el cuidado a los derechos de la ciudadanía y las familias. En particular, las mujeres siguen asumiendo una carga que se acrecienta con el aumento de las personas dependientes. Cómo organizar de manera socialmente responsable y equitativa el cuidado es uno de los retos más importantes a los que tendremos que enfrentarnos como sociedad si queremos garantizar la continuidad y la calidad de vida de la población.

\section{Referencias}

Barker, Drucilla y Feiner, Susan (2010), "As the world turns: Globalization, consumption, and the feminization of work", en Rethinking Marxism, núm. 22, vol. 2, Estados Unidos: Routledge, Taylor \& Francis. Disponible en: http://www.tandfonline.com/doi/ abs/10.1080/08935691003625521 [10 de julio de 2016].

Batthyamy, Karina (2009), “Género, cuidados familiares y uso del tiempo”, en Aguirre, Rosario [ed.], Las bases invisibles del bienestar social. El trabajo no remunerado en Uruguay, Uruguay: Unifem Uruguay. Disponible en: http://www.inju.gub.uy/innovaportal/ file/21713/1/2_las_bases_invisibles_del_bienestar_social.pdf [21 de mayo de 2016]. 
Becker, Gary (1987), Tratado sobre la familia, España: Alianza.

Bedford, Katey y Shirin, Rai (2010), "Feminists theorize international political economy", en Signs, núm. 36, vol. 1, Estados Unidos: The University of Chicago Press. Disponible en: http://www.jstor.org/stable/10.1086/652910 [10 de mayo de 2016].

Benería, Lourdes (1979), "Reproduction, production and the sexual division of labour", en Cambridge Journal of Economics, núm. 3, vol. 3, Reino Unido: Oxford University Press.

Bonino, Luis (2001), "Los varones hacia la paridad en lo doméstico, discursos sociales y prácticas masculinas”, en Sánchez-Palencia, Carolina e Hidalgo, Juan-Carlos [eds.], Masculino plural: construcciones de la masculinidad, España: Universidad de Lleida. Disponible en: http://www.luisbonino.com/pdf/Los\%20varones\%20ante\%20el\%20 problema\%20de\%20la\%20igualdad\%20con\%20\%20las\%20mujeres.pdf [3 de septiembre de 2016].

Carrasco, Cristina (2011), "La economía del cuidado: planteamiento actual y desafíos pendientes”, en Revista de Economía Crítica, núm. 11, España: Asociación Cultural Economía Crítica. Disponible en: http://www.revistaeconomiacritica.org/sites/default/files/revistas/n11/REC11_9_intervenciones_CristinaCarrasco.pdf [22 de junio de 2016].

Carrasco, Cristina (2013), "El cuidado como eje vertebrador de una nueva economía”, en Cuadernos de Relaciones Laborales, núm. 31, España: Ediciones Complutense. Disponible en: http://revistas.ucm.es/index.php/CRLA/article/view/41627 [15 de junio de 2016].

Daly, Mary y Lewis, Jane (2000), “The concept of social care and the analysis of contemporary welfare states”, en British Journal of Sociology, núm. 51, Inglaterra: London School of Economics.

Dema-Moreno, Sandra y Díaz-Martínez, Capitolina (2014), "Los sesgos de género en las encuestas oficiales sobre economía doméstica”, en Revista Española de Investigaciones Sociológicas, núm. 148, España: Centro de Investigaciones Sociológicas. Disponible en: http://dx.doi.org/10.5477/cis/reis.148.21 [27 de marzo de 2017].

Díaz-Fernández, Montserrat y Llorente-Marrón, Mar (2011), "Estimaciones de la demanda mundial de cuidado, 2010-2050. Una aproximación econométrica”, en Documentos de Trabajo BBVA, España: Fundación BBVA. Disponible en: http://www.fbbva.es/ $\mathrm{TLFU} / \mathrm{tlfu} / \mathrm{esp} / \mathrm{publicaciones/documentos/fichadoc/index.jsp?codigo=656} \mathrm{[23 \textrm {de }}$ junio de 2013].

Durán, María-Ángeles (2006), "Dependientes y cuidadores. El desafío de los próximos años", en Revista del Ministerio de Trabajo y Asuntos Sociales, núm. 60, España: Ministerio de Trabajo y Asuntos Sociales. Disponible en: http://www.empleo.gob.es/es/publica/ pub_electronicas/destacadas/revista/numeros/60/Est04.pdf [13 de junio de 2016].

Ellingstaeter, Anne (1999), "Dual Breadwinners between State and Market", en Crompton, Rosemary (ed.), Restructuring gender relations and employment. The decline of the male breadwinner, Estados Unidos: Oxford University Press.

Enríquez, Corina (2012), “Care: the missing link in economic analysis?", en Revista CEPAL, núm. 106, Chile: CEPAL. Disponible en: http://repositorio.cepal.org/handle/11362/11580 [10 de mayo de 2016].

Esquivel, Valeria (2011), "La Economía del Cuidado en América Latina: poniendo a los cuidados en el centro de la agenda", en Cuadernos Atando Cabos; deshaciendo nudos, núm. 2, Panamá: Centro Regional de América Latina y el Caribe del PNUD. Disponible 
Montserrat Díaz-Fernández, Mar Llorente-Marrón y Sandra Dema-Moreno. Tratamiento económico y modelización del cuidado

en: http://192.64.74.193/ genera/es/documentos/Atando_Cabos.pdf [11 de mayo de 2016].

Ferrant, Gaëlle et al. (2014), "Unpaid Care Work: The missing link in the analysis of gender gaps in labour outcomes", en OECD Development Center, Francia: OCDE. Disponible en: https://www.oecd.org/dev/development-gender/Unpaid_care_work.pdf [1 de octubre de 2017].

Folbre, Nancy (1995), "Holding Hands at Midnight: The Paradox of Caring Labor", en Feminist Economics, núm. 1, Estados Unidos: Routledge. Disponible en: http://cscs. res.in/dataarchive/textfiles/textfile.2008-08-28.7346961453/file [6 de mayo de 2016].

Folbre, Nancy (2006), "Measuring Care: Gender, Empowerment, and the Care Economy", en Journal of Human Development, núm. 7, vol. 2, Estados Unidos: Routledge. Disponible en: https://www.amherst.edu/media/view/92075/.../measuring\%2Bcare.pdf [20 de junio de 2016].

Folbre, Nancy (2015), "Valuing Non-market Work", en UNDP Human Development Report Office, Estados Unidos: UNDP. Disponible en: http://hdr.undp.org/es/content/valuing-non-market-work [6 de mayo de 2016].

Frazis, Harley y Stewart, Jay (2011), "How Does Household Production Affect Measured Income Inequality?”, en Journal of Population Economics, núm. 24, Estados Unidos: Springer. Disponible en: https://doi.org/10.1007/s00148-009-0258-3 [6 de mayo de 2016].

Frick, Joachim et al. (2009), “The Impact of Home Production on Economic Inequality in Germany”, en Empirical Economics, núm. 43, Estados Unidos: Springer. Disponible en: http://dx.doi.org/10.1007/s00181-011-0523-7 [6 de mayo de 2016].

Gardiner, Jean (1997), Gender, Care and Economics, Inglaterra: MacMillan Press.

García-Calvente, María del Mar et al. (2004), "El impacto de cuidar la salud y la calidad de vida de las mujeres”, en Gaceta Sanitaria, núm. 18, España: Sociedad Española de Salud Pública y Administración Sanitaria. Disponible en: http://www.gacetasanitaria.org/ es/el-impacto-cuidar-salud-calidad/articulo/13061998/ [2 de septiembre de 2016].

Gutiérrez-Rodríguez, Encarnación (2014), "Domestic work-affective labor: On feminization and the coloniality of labor", en Women's Studies International Forum, núm. 46, Reino Unido: Elsevier. Disponible en: http://dx.doi.org/10.1016/j.wsif.2014.03.005 [14 de mayo de 2016].

Himmelweit, Susan (1995), “The Discovery of 'Unpaid Work': The Social Consequences of the Expansion of Work”, en Feminist Economics, núm. 1, Estados Unidos: Routledge.

Himmelweit, Susan (2002), "Making Visible the Hidden Economy: The Case for GenderImpact. Analysis of Economic Policy”, en Feminist Economics, núm. 8, Estados Unidos: Routledge.

Himmelweit, Susan (2007), “The prospects for caring: Economic theory and policy analysis”, en Cambridge Journal of Economics, vol. 31 núm. 4, Reino Unido: Oxford University Press.

Izquierdo, María Jesús (2003), "Del sexismo y la mercantilización del cuidado a su socialización: hacia una política democrática del cuidado", comunicación presentada en el Congreso Internacional SARE 2003 "Cuidar cuesta: costes y beneficios del cuidado”, España: Emakunde. Disponible en: http://www.sidocfeminista.org/images/ books/04225/04225_00.pdf [9 de julio de 2016]. 
Kofman, Eleanor (2012), "Rethinking care through social reproduction: Articulating circuits of migration”, en Social Politics, núm. 19, Reino Unido: Oxford Journals. Disponible en: http://sp.oxfordjournals.org/content/early/2012/01/18/sp.jxr030.full. pdf + html [19 de junio de 2016].

Lewis, Jane (2007), “Gender, Ageing and the 'New Social Settlement': The importance of developing a holistic approach to care policies”, en Current Sociology, núm. 55, Reino Unido: Sage. Disponible en: http://csi.sagepub.com/content/55/2/271.short [6 de septiembre de 2016].

McMahon, Anthony (1999), Taking Care of men, Reino Unido: Cambridge University Press.

OIT (Organización Internacional del Trabajo) (2016), Perspectivas sociales y del empleo en el mundo. Tendencias 2016, Suiza: OIT. Disponible en: http://www.ilo.org/global/ research/global-reports/weso/2016/lang--es/index.htm [6 de julio de 2016].

Pérez-Orozco, Amaia (2004) "Estrategias feministas de deconstrucción del objeto de estudio de la economía”, en Foro Interno: Anuario de Teoría Politica, núm. 4, España: Universidad Complutense de Madrid. Disponible en: http://pendientedemigracion. ucm.es/info/ec/jec9/pdf/A05\%20-\%20P\%E9rez\%20Orozco,\%20Amaia.pdf [8 de julio de 2016].

Pérez-Orozco, Amaia (2009), "Global perspectives on the social organization of care in times of crisis: assessing the situation", en Gender, Migration and Development, Working Paper, núm. 5, Estados Unidos: UN-INSTRAW. Disponible en: http:// www.flacsoandes.org/generoycultura/Publicaciones/Publicacionesprofesoras/Profesorasasociadas/Perez-Orozco Amaia/Papers/Amaia_Perez_Orozco_Working_Paper_6\%20ingles.pdf [18 de julio de 2016].

Peterson, Spike (2009), "Interactive and intersectional analytics of globalization", en Frontiers: A Journal of Women Studies, núm. 30, vol. 1, Estados Unidos: Taylor \& Francis.

Picchio, Antonella (1996), "The Analytical and Political Visibility of the work of Social Reproduction”, en Background Papers, Human Development Report 1995, Estados Unidos: UNDP. Disponible en: http://hdr.undp.org/sites/default/files/reports/256/ hdr_1995_en_complete_nostats.pdf [14 de mayo de 2016].

Picchio, Antonella (1999), "Visibilidad analítica y política del trabajo de reproducción social", en Carrasco, Cristina [ed.], Mujeres y Economia, España: Icaria.

Picchio, Antonella (2001), “Un enfoque macroeconómico 'ampliado' de las condiciones de vida”, conferencia inaugural de las Jornadas “Tiempos, trabajos y género”, España: Facultad de Ciencias Económicas, Universidad de Barcelona. Disponible en: www1.paho. org/Spanish/HDP/hdw/chile-pi.PDF [14 de mayo de 2016].

Picchio, Antonella (2009), "Condiciones de vida: Perspectivas, análisis económico y políticas públicas”, en Revista de Economía Crítica, núm. 7, España: Asociación Cultural Economía Crítica. Disponible en: www.revistaeconomiacritica.org [6 de junio de 2016].

Ricardo, David (2003), Principios de Economía Politica y Tributación, España: Ediciones Pirámide.

Rodríguez-Cabrero, Gregorio (2007), “La protección social de la dependencia en España. Un modelo sui generis en el desarrollo de los derechos sociales", en Politica y Sociedad, núm. 44, vol. 2, España. Disponible en: http://revistas.ucm.es/index.php/POSO/article/viewFile/POSO0707230069A/22257 [20 de febrero de 2017]. 
Rogero, Jesús (2010), Los tiempos del cuidado. El impacto de la dependencia de los mayores en la vida cotidiana de sus cuidadores, España: Instituto de Mayores y Servicios Sociales (IMSERSO).Disponible en: http://www.uam.es/personal_pdi/economicas/jrogero/ docs/Rogero_Garcia_10.pdf [15 de febrero de 2017].

Smith, Adam (2011), La riqueza de las Naciones, España: Alianza.

Tobío, Constanza et al. (2010), "El cuidado de las personas. Un reto para el siglo XXI", en Colección Estudios Sociales, núm. 28, España: Obra Social Fundación la Caixa. Disponible en: https://multimedia.caixabank.es/lacaixa/ondemand/obrasocial/pdf/estudiossociales/vol28_completo_es.pdf [3 de mayo de 2016].

Yoon, Jayoung (2014), "Counting care work in social policy: Valuing unpaid child and elder care in Korea”, en Feminist Economics, núm. 20, Estados Unidos: Routledge.

\section{Anexo}

\section{Figura 1}

\section{El equilibrio del mercado de cuidado}

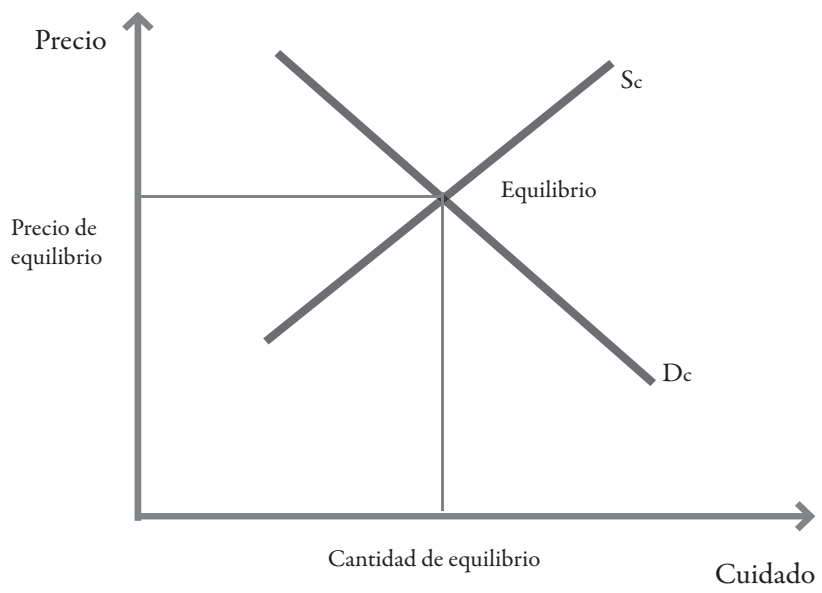

Fuente: Elaboración propia.

Montserrat Díaz-Fernández. Doctora por la Universidad de Oviedo (España) y Catedrática de la Universidad en el Departamento de Economía Cuantitativa de la Universidad de Oviedo. Líneas de investigación: Modelización econométrica del fenómeno demográfico (fecundidad, aborto, cuidado y envejecimiento) y mercado de la vivienda (necesidades de vivienda, equilibrio del mercado, oferta y demanda de vivienda). Publicaciones recientes: 
Díaz-Fernández, Montserrat y Llorente-Marrón, Mar, "Estimaciones de la demanda mundial de cuidado, 2010-2050. Una aproximación econométrica”, en Documentos de Trabajo BBVA, Madrid: Fundación BBVA (2011). Disponible en: http://www.fbbva.es/TLFU/tlfu/esp/publicaciones/documentos/fichadoc/index.jsp?codigo=656; Llorente-Marrón, Mar y DíazFernández, Montserrat, "Fecundidad y estructura familiar en España”, en Interciencia, núm. 11, Caracas, Venezuela: Asociación Interciencia (2014). Disponible en: http://www.redalyc.org/articulo.oa?id=33932572002; Díaz-Fernández, Montserrat y Llorente-Marrón, Mar y Paz Méndez-Rodríguez, "¿Condiciona la población la dinámica de la actividad inmobiliaria? Un análisis de cointegración para el caso español”, en Investigación Económica, núm. 75, México: Elservier (2016). Disponible en: http://www.elsevier.es/ es-revista-investigacion-economica-122-articulo-condiciona-poblaciondinamica-actividad-inmobiliaria-S0185166716300303.

Mar Llorente-Marrón. Doctora por la Universidad de Oviedo (España) y Profesora Titular de Economía Cuantitativa de la Universidad de Oviedo. Líneas de investigación: economía de la población, econometría aplicada y evaluación del impacto del proceso demográfico en la economía, brecha salarial, política de género. Publicaciones recientes: Díaz-Fernández, Montserrat y Llorente-Marrón, Mar, "Estimaciones de la demanda mundial de cuidado, 2010-2050. Una aproximación econométrica”, en Documentos de Trabajo BBVA, Madrid: Fundación BBVA (2011). Disponible en: http://www. fbbva.es/TLFU/tlfu/esp/publicaciones/documentos/fichadoc/index. jsp?codigo=656; Llorente-Marrón, Mar y Díaz-Fernández, Montserrat, "Fecundidad y estructura familiar en España”, en Interciencia, núm. 11, Caracas, Venezuela: Asociación Interciencia (2014). Disponible en: http:// www.redalyc.org/articulo.oa?id=33932572002; Díaz-Fernández, Montserrat y Llorente-Marrón, Mar y Paz Méndez-Rodríguez, "Contextual determinants of induced abortion: a panel analysis”, en Revista de Saúde Pública, núm. 50, São Paulo, Brasil: Faculdade de Saúde Pública da Universidade de São Paulo (2016). Disponible en: http://www.scielo.br/pdf/rsp/v50/0034-8910-rspS1518-87872016050005917.pdf

Sandra Dema-Moreno. Doctora por la Universidad de Oviedo (España) y Profesora Titular de Sociología en la citada Universidad. Líneas de investigación: análisis de las políticas públicas de igualdad, las desigualdades entre hombres y mujeres en el manejo del dinero, la participación laboral de las mujeres y la integración de la perspectiva de género en 
la cooperación al desarrollo. Publicaciones recientes: Díaz-Martínez, Capitolina, Dema-Moreno, Sandra y Lucila Finkel, "Desigualdades de género en la distribución de recursos económicos en las parejas españolas”, en Papers, Revista de Sociología, núm. 100, Madrid, España: Consejo Superior de Investigaciones Científicas (2015). doi: http://dx.doi.org/10.5565/rev/papers.591; Dema-Moreno, Sandra y Díaz-Martínez, Capitolina, "Los sesgos de género en las encuestas oficiales sobre economía doméstica”, en Revista Española de Investigaciones Sociológicas, núm. 148, Madrid, España: Centro de Investigaciones Sociológicas (2014). doi: http://dx.doi.org/10.5477/ cis/reis.148.21; Díaz-Martínez, Capitolina y Dema-Moreno, Sandra [eds.], Sociología y Género, Madrid: Tecnos (2013).

Fecha de recepción: 31 de marzo de 2017.

Fecha de aprobación: 03 de octubre de 2017. 
